\title{
Mental Deficiency as a Basic Discipline in the Training of a Psychiatry"
}

By ROBERT GIBSON, M.D., Ch.B., D.P.M. (Dundee, Scotland) (Consultant Psychiatrist, Scottish Eastern Hospital Region; Lecturer in Mental Deficiency, University of St. Andrews)

The field of mental deficiency is one with which the psychiatrist is not always familiar. By the time he has obtained his training in the psychoses and neuroses, explored psychosomatic medicine, and spread his wings towards the horizon of social psychiatry, too often the opportunity is neglected to gain a full experience in this particular field. Mental deficiency has been described as the Cinderella of psychiatry. The simile is apt, not merely in the opening scene, but also in the denouement, as is soon apparent to those who tarry longer.

The number of defectives in the community has been variously estimated, and is probably about $2 \%$. This is a considerable figure, and even if we refrain from adding the much larger borderline group the magnitude of the subject is obvious. Its significance to psychiatry is likewise becoming more apparent, and it is hardly a coincidence that in Britain candidates for the diploma in this specialty are being increasingly required to undergo a period of training in a mental deficiency institution.

By its very nature mental deficiency has firm connections with general medicine and genetics, whilst its ramifications extend into the kindred fields of education and sociology. It thus offers on the one hand a close bond with the basic disciplines of medicine, and at the same time through its wide contacts in the educational sphere it opens to the embryo psychiatrist a more sweeping vista and a broader conception of the field he is setting out to cultivate. To such a one, early in his career, mental deficiency is well placed to offer that contact with the ground that may yet serve to keep his feet firmly on the path as he gropes his way through the mist of psychological speculation.

To this end the mental deficiency institution can bring forward its wards, its school and training centres, and its clinics.

In the wards will be found a wide array of cases whose intrinsic merit ranges from those almost purely medical to others capable of taxing the ingenuity of the experienced psychiatrist. It is sometimes forgotten that mental defect is associated with at least 70 different conditions, some exceedingly rare. Thus the student will encounter the common subcultural defective, representing the lower end of normal variation of the population, and the groups where amentia is associated with behaviour disorders, congenital syphilis,

* Reprinted from the American Journal of Psychiatry, July 1951, by permission of the Editor. 
mongolism, endocrine dystrophies, skeletal, neuromuscular, and cutaneous conditions, and abnormalities of the special sense organs. In those with behaviour disorders most institutions can offer cases of amentia due to brain-injury, epileptic amentia, and even frank psychosis such as infantile schizophrenia. Indeed the knowledge gained from this group alone can pave the way to the investigation of childhood psychoses, whilst detailed studies in the psychopathology of the brain-injured child can simplify later the understanding of the brain-injured adult. The skeletal group includes about 20 conditions, mostly rare, ranging from microcephaly to acrocephalosyndactyly, but it is in the neuromuscular group that close contact is made with neurology, and here the mental deficiency institution is often able to supplement the instruction in this subject...

There is also considerable scope for bio-chemical studies, and one need only instance phenylpyruvic oligophrenia with its failure to metabolize phenylalanine. In like fashion the influence of genetics is abundantly clear in mental defect, but it is not so commonly realised that environmental factors also have a part to play, and that along this little-trodden path information can be culled which may throw further light on personality structure and function.

It has been shown by Wechsler, Rappaport, and others that psychometrics, for long almost the monopoly of mental deficiency, can further the more exact diagnosis of the psychoses and neuroses, and it is probably true that of all branches of psychiatry mental deficiency is best equipped to give a fundamental training in mental testing.

It is increasingly recognised that the diagnosis of the higher grades of mental defect as well as of borderline defect is not the simple procedure it was once thought to be. In establishing a satisfactory diagnosis it may indeed be necessary to apply a series of tests, verbal and performance, social maturity, and perhaps, in some cases, projective techniques as well. A thorough training can thus be had in a comprehensive range of tests, such as the TermanMerrill, Wechsler-Bellevue, Progressive Matrices, and others purely performance, whilst more specialised tests of memory can be applied, and some instruction obtained in achievement and vocational tests. Moreover, by a study of the test pattern obtained from the thorough examination of a case useful pointers can be gathered on the differential aspects of mental development, from concept formation to concentration, and on the selective impairment of these aspects. One need hardly cite the crippling effect of anxiety on the digit-span subtest of the Wechsler-Bellevue Scale, or the impairment and severe distortion revealed by Babcock's Learning Efficiency Test in suspected schizophrenia, in order to realise the advantage of a basic training in mental testing. 
As mental defect may be complicated by psychopathy, psychosis, or psychoneurosis, some experience can be gained in such conditions. Although mild manic-depressive phases are not uncommon, a more useful introduction to the psychoses will be found in epilepsy and more especially in the schizophrenia that may be superimposed on feeble-mindedness. Indeed, schizophrenic features are probably those most prevalent in this group, including the interesting primitive catatonic psychosis occurring in mongols. Psychoneuroses occur more often in the dull and defective than in the normal population, and it is not long before the worker in this field comes across anxiety states and hysteria, in the latter event not rarely encountering such gross manifestations as hysterical fits or psychogenic delirium. Again in the differential diagnosis further consideration must be given to psychopathy, schizophrenia simplex, as well as to a number of special disabilities ranging from impaired hearing to congenital word-blindness, which are capable of lowering the patient's reaction level.

The training and educational functions of the mental deficiency institution are almost a chapter in themselves. They range from the simplest of occupational therapy to undertaking preliminary training for industry, whilst the school work includes sensory training, speech therapy, and the methods applicable to braininjured children.

Through its mentally-handicapped clinics the institution affords the trainee psychiatrist opportunity to see mental deficiency against the background of psychiatry as a whole, for to these clinics will be sent feebleminded and borderline defectives of all types, whether in pure culture or complicated by psychosis, psychoneurosis, or psychopathy, to receive investigation from the diagnostic, forensic, or vocational aspects.

Finally, treatment in mental deficiency is receiving increasing attention. Apart from such simple procedures as the administration of thyroid or glutamic acid, more drastic treatments like prefrontal leucotomy are showing promise. The latter operation is of value in high-grade cases where psychopathy is a complication and where behaviour disorder generally is a marked feature, whilst recently McKhann has described the results of his operation for the revascularisation of the brain in chosen cases. Electroshock methods are likewise not without their uses, with indications similar to those in general psychiatry. Psychotherapy in mental deficiency has been largely neglected, yet it is apparent that the need for this does not vanish as the IQ drops below 70, and the increasing realisation that the defective has a personality as well as an intelligence quotient is likely to be accompanied by a development of this field. Indeed, from a survey of factors such as these it may well be that the time is not far distant when the accent will be on the dynamic rather than the static aspects of mental deficiency. 